\title{
Commentary: Patient Safety in the Home
}

Shirlee Sharkey and Helene Lacroix

$\mathrm{I}$ n 2018, SE Health (formerly Saint Elizabeth Health Care) delivered care and support to more than 500,000 people when and where they needed it. We delivered many of these health services to Canadians in their own homes, supporting independent living and freeing up hospital beds for acute care needs. As care in one's home becomes more commonplace, it is critical that Canadians know they are safe.

In addition to knowing that effective care is being delivered to facilitate health and wellness, it is imperative that Canadians have the safest homecare experience possible. So far, most patient safety work has focused on facility-based settings such as hospitals and long-term care homes. There is an ongoing need to not only translate and adapt these learnings but also to further develop our own understanding of and approaches to enhancing patient safety in the home.

There are unique safety risks for providers, patients and family caregivers in the home setting, including physical, environmental and social factors. Unlike institutional environments that are highly regulated and controlled, the home is the domain of the patient - it reflects the diverse ways people live and may not always be optimally designed for the delivery of healthcare. Patient safety in the home therefore requires us to work collaboratively with patients and their families to identify, manage and minimize risks as much as possible.

As part of its Home Care Safety Roundtable Action Plan, the Canadian Patient Safety Institute commissioned researchers from SE Health to conduct an environmental scan for available resources that improve communication between interdisciplinary healthcare providers and organizations involved in transitioning patients into and out of homecare. This list of more than 50 forms, communication guides, information technology systems and software is available from the SE Research Centre.

Tools aside, the critical first step is to ensure a safe system of care in the home environment. The first line of safety is supporting the proficiency of the patient and the family to effectively manage the health condition at home. This is enabled by the next line of safety: the workforce that interacts with patients every day. The world of human factors tells us that people's personalities, actions, skills and competencies are variable and susceptible to error, especially in poorly designed workplaces. This holds true in every industry, and since we cannot change the human condition, we have to change the conditions under which humans work.

We have to be willing and determined to cultivate a system with multiple layers of defense - many safety nets, if you will. In addition to investing in education and training (which improves an individual's competency), we can use standardized tools, such as the International Resident Assessment Instrument (InterRAI), to reduce variability in care planning and to monitor outcomes. We also have to commit to continually reviewing professional practice and organizational 
culture to identify preconditions for error and intervene before an adverse event occurs.

If we take these actions, we will lay a solid foundation for a "just culture of patient safety" and learning in homecare. What does that look like? It's a culture in which we move beyond incident reporting to proactively seek out and understand the factors that contribute to both risk and safety in the uncontrolled home environment and then devise strategies to address these factors at all levels of care and in all interactions. It's also about moving beyond blame, beyond the current reflexive response of punitive investigations of adverse events. It's about reaching outside the healthcare box and embracing systems thinking and design as a response to adverse events.

Over the past decade, the homecare sector has made strides in many of these areas. We must be relentless in continuing this focus, but we cannot do it alone. Health system support and ongoing collaboration with patients and families are required to optimize safety in the environments in which we live and work. There is no finish line when it comes to patient safety. Like high-quality and person-centred care, it's an ongoing journey.

We know how to do better and safer work in hospitals and long-term care facilities, and we can do the same at home. HQ

\section{About the Authors}

Shirlee Sharkey, BA, BScN, MHSc, CHE, ICD.D, is the chief executive officer of SE Health, a national social enterprise bringing excellence and innovation to homecare, seniors living and family caregiving. She is a member of the Premier's Council on Improving Healthcare and Ending Hallway Medicine, and serves on numerous boards including the CD Howe Institute and University Health Network

Helene Lacroix, RN, BScN, MSc, is the vice president of clinical innovation at SE Health. Throughout her career she has worked to elevate quality and safety in healthcare and played an active role in spreading best practices on a national scale. She was part of the research team that led a landmark pan-Canadian homecare safety study. 


\section{SOINS À DOMICILE ET SÉCURITÉ DES PATIENTS}

\section{Commentaire : la sécurité des patients à domicile}

Shirlee Sharkey et Helene Lacroix

$\mathrm{E}$ n 2018, l'organisme de santé SE Health (anciennement Saint Elizabeth Health Care) a fourni des services à plus de 500000 personnes au moment et à l'endroit où elles en avaient besoin. Plusieurs de ces services de santé ont été fournis à domicile, préservant ainsi l'autonomie des usagers tout en libérant des lits d'hôpitaux pour d'autres besoins en soins de courte durée. Avec de plus en plus de soins fournis à domicile, il est essentiel que les Canadiens se sentent en sécurité.

En plus de soins efficaces fournis pour faciliter la santé et le mieux-être, il est impératif que les Canadiens bénéficient des soins à domicile les plus sécuritaires possible. Jusqu'à présent, la plupart des efforts pour améliorer la sécurité des patients ont plutôt concerné les établissements comme les hôpitaux et les centres de soins de longue durée. Maintenant, il faut non seulement intégrer et adapter ces apprentissages, mais également développer nos démarches et notre perception du problème dans le but d'améliorer la sécurité des patients dans le contexte des soins à domicile.

Les soins à domicile comportent leur propre lot de risques pour les prestataires, les patients et les proches aidants. Ces risques concernent des facteurs physiques, environnementaux et sociaux. Contrairement aux environnements institutionnels fortement réglementés et contrôlés, le lieu de résidence est le domaine du patient - il véhicule la façon de vivre des personnes et n'est pas toujours conçu pour une prestation optimale des services de santé. La sécurité des patients à la maison exige donc un travail en collaboration avec les patients et leurs familles pour identifier, gérer et minimiser les risques autant que possible.

Dans le cadre du Plan d'action de la table ronde sur la sécurité des soins à domicile, l'Institut canadien pour la sécurité des patients a chargé des chercheurs de SE Health de procéder à une analyse de l'environnement pour recenser les ressources disponibles susceptibles d'améliorer la communication entre les prestataires de services de santé interdisciplinaires et les organisations responsables des transitions pour les patients requérant des soins à domicile. Cette liste de plus de 50 formulaires, guides de communication, systèmes informatiques et logiciels est disponible auprès du Centre de recherche de SE Health.

Au-delà des outils, la première étape essentielle est d'assurer un système de soins sécuritaire adapté à l'environnement du domicile. Le premier pas en matière de sécurité est d'assurer au patient et à sa famille un soutien pour la gestion de l'état de santé à la maison. Cette étape est effectuée par le biais d'interactions quotidiennes du personnel avec les patients. La réalité des facteurs humains nous apprend que la personnalité des gens, leurs actions, leurs qualifications et leurs compétences varient beaucoup et peuvent devenir des sources d'erreurs, tout particulièrement dans les lieux de travail moins bien adaptés. C'est vrai pour tous les secteurs, et puisque nous ne pouvons pas changer la condition humaine, nous devons modifier les 
conditions dans lesquelles les humains travaillent.

Il faut avoir la volonté et la détermination de mettre en place un système qui comporte plusieurs niveaux de défense - de nombreux filets de sécurité, si vous préférez. En plus d'investir dans l'éducation et la formation (ce qui améliore les compétences d'un individu), il est possible d'employer des outils normalisés, tels que le système international d'évaluation des résidents (InterRAI), pour réduire la variabilité dans la planification des soins et surveiller les résultats. Il faut également s'engager à réexaminer régulièrement les pratiques professionnelles et la culture organisationnelle afin d'identifier les conditions préalables à l'erreur et d'intervenir avant qu'un événement indésirable ne se produise.

Si nous adoptons ces mesures, nous jetterons les bases d'une " culture équitable " en matière de sécurité des patients et d'apprentissage dans la prestation des soins à domicile. Comment cela se traduit-il dans la pratique? Cela devient une culture où nous allons au-delà de la simple déclaration d'incidents, où nous détectons et cherchons à comprendre de manière proactive les facteurs pouvant contribuer aux risques et à la sécurité dans un environnement domestique non contrôlé, puis élaborer des stratégies pour tenir compte de ces facteurs à tous les niveaux de soins et dans toutes les interactions. Il faut aussi passer outre le blâme, l'automatisme des réponses actuelles comportant des pratiques punitives suite aux événements indésirables. L'idée est de sortir des sentiers battus dans le domaine des soins de santé en adoptant un système pensé et conçu en fonction de la prévention des événements indésirables.

Au cours des 10 dernières années, le secteur des soins de santé à domicile a progressé dans bon nombre de ces domaines. Il faut poursuivre cet objectif, mais nous ne pouvons y arriver seuls. Le soutien du système de santé et la collaboration continue avec les patients et leurs familles sont nécessaires pour optimiser la sécurité dans les environnements où nous évoluons et travaillons. Il n'y a pas de ligne d'arrivée en ce qui concerne la sécurité des patients. Tout comme les soins de haute qualité axés sur la personne, c'est une démarche qui n’arrête jamais.

Nous savons comment mieux effectuer notre travail de façon sécuritaire dans les hôpitaux et les établissements de soins de longue durée, et nous pouvons faire la même chose à la maison. HQ

\section{Au sujet des auteures}

Shirlee Sharkey, B.A., B.Sc.inf., M.Sc., CHE, LLD (honorifique), est directrice générale de SE Health, une entreprise sociale nationale apportant excellence et innovation aux personnes âgées et aux familles dans les soins à domicile. Elle est membre du Conseil du premier ministre pour l'amélioration des soins de santé et l'élimination de la médecine de couloir et siège à de nombreux autres conseils, dont ceux de I'Institut CD Howe et University Health Network.

Helene Lacroix, I.A., B.Sc.inf., M.Sc., est vice-présidente à I'innovation clinique chez SE Health. Au cours de sa carrière, elle a œuvré pour améliorer la qualité et la sécurité des soins de santé et a joué un rôle actif dans la diffusion de meilleures pratiques à l'échelle nationale. Elle faisait partie de l'équipe de recherche qui a dirigé une étude pancanadienne sur la sécurité des soins à domicile. 